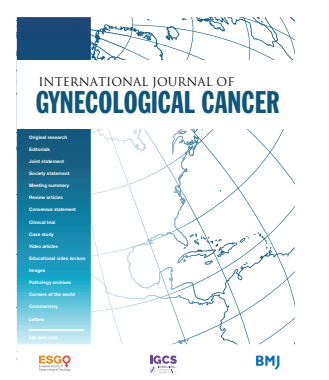

\title{
Oncologic outcomes of minimally invasive versus open radical hysterectomy for early stage cervical carcinoma and tumor size $\leq 2$ cm: a systematic review and meta-analysis
}

Dimitrios Nasioudis (D) ," Benjamin B Albright (D) , ${ }^{2}$ Emily M Ko, ${ }^{1}$ Ashley F Haggerty, Robert L Giuntoli II, ${ }^{1}$ Sarah H Kim, ${ }^{1}$ Mark A Morgan, ${ }^{1}$ Nawar A Latif ${ }^{1}$

- Additional supplemental material is published online only. To view, please visit the journal online (http://dx.doi.org/ 10.1136/ijgc-2021-002505).

${ }^{1}$ Division of Gynecologic Oncology, Penn Medicine, Philadelphia, Pennsylvania, USA ${ }^{2}$ Division of Gynecologic Oncology, Duke University, Durham, North Carolina, USA

Correspondence to Dr Dimitrios Nasioudis, Department of Obstetrics and Gynecology, Hospital of the University of Pennsylvania, Philadelphia 19104, Pennsylvania, USA; dimitrios. nasioudis@uphs.upenn.edu

Received 7 February 2021 Revised 26 April 2021 Accepted 28 April 2021 Published Online First 20 May 2021
Check for updates

(C) IGCS and ESGO 2021. № commercial re-use. See rights and permissions. Published by BMJ.

To cite: Nasioudis $D$, Albright BB, Ko EM, et al. Int J Gynecol Cancer 2021;31:983990.

\section{HIGHLIGHTS}

- Minimally invasive radical hysterectomy for cervical cancer and tumor size $\leq 2 \mathrm{~cm}$ is associated with worse progressionfree survival.

- Patterns of relapse appear comparable; rate of distant relapse was comparable between the two groups.

- Minimally invasive radical hysterectomy should be avoided for all patients with cervical cancer despite tumor size.

\section{ABSTRACT}

Objective To investigate the oncologic outcomes of patients with early-stage cervical carcinoma and tumor size $\leq 2 \mathrm{~cm}$ who underwent open or minimally invasive radical hysterectomy.

Methods The Pubmed/Medline, Embase, and Web-ofScience databases were queried from inception to January 2021 (PROSPERO CRD 42020207971). Observational studies reporting progression-free survival and/or overall survival for patients who had open or minimally invasive radical hysterectomy for early-stage cervical carcinoma and tumor size $\leq 2 \mathrm{~cm}$ were selected. Level of statistical heterogeneity was evaluated with the $\mathrm{I}^{2}$ statistic. A randomeffects model was used to compare progression and overall survival between the two groups and HR with 95\% confidence intervals were calculated with the Der Simonian and Laird approach. Risk of bias and quality of included studies was assessed with the Newcastle-Ottawa scale.

Results A total of 10 studies that met the inclusion criteria were included encompassing 4935 patients. Of these, $2394(48.5 \%)$ patients had minimally invasive and 2541 (51.5\%) patients had open radical hysterectomy; respectively. Patients who underwent minimally invasive hysterectomy had worse progression-free survival than those who had open surgery (HR 1.68, 95\% $\mathrm{Cl} 1.20,2.36$, $\mathrm{I}^{2} 26 \%$ ). Based on five studies, patients who had minimally invasive $(n=1808)$ hysterectomy had a trend towards worse overall survival than those who had open surgery $(\mathrm{n}=1853)$ (HR 1.64, 95\% Cl 1.00 to 2.68, $\left.\left.\right|^{2} 15 \%\right)$.

Conclusion Based on a systematic review of the literature and meta-analysis of studies that control for confounders, for patients with cervical cancer and tumor size $\leq 2 \mathrm{~cm}$, minimally invasive radical hysterectomy was associated with worse progression-free survival than laparotomy.

\section{INTRODUCTION}

Cervical cancer is the fourth most common gynecologic malignancy worldwide. ${ }^{1}$ For patients with apparent FIG0 2009 stage IA1 with lymphovascular invasion/IA2-IB2 disease, radical hysterectomy with lymphadenectomy or sentinel lymph node biopsy is recommended. ${ }^{2}$ Data from retrospective studies show that minimally invasive hysterectomy can be associated with lower blood loss, decreased rate of post=operative complications, faster recovery, and shorter inpatient hospital stay. ${ }^{3}$ However, the widespread use of minimally invasive surgery for patients with cervical cancer was adopted without evidence from randomized trials and was based on small retrospective studies with a relatively short follow-up. ${ }^{3}$ A recent randomized trial demonstrated worse progression-free survival for patients who had minimally invasive radical hysterectomy. ${ }^{4}$ In addition, an analysis of the National Cancer Database also revealed inferior overall survival for a similar patient population who underwent minimally invasive radical hysterectomy between 2010 and $2013 .{ }^{5}$ It is unclear whether a higher relapse rate is related to the use of uterine manipulator, spread of tumor cells during colpotomy, or inadequate surgical technique. ${ }^{6} \mathrm{~A}$ recent meta-analysis of high-quality observational studies also concluded that there is an increased risk of relapse among patients undergoing minimally invasive radical hysterectomy, however a sub-analysis based on tumor size was not performed. ${ }^{6}$

While major organizations such as FIG0, European Society of Gynecological Oncology, Society of Gynecologic Oncology, National Comprehensive Cancer Network guidelines discourage the performance of minimally invasive radical hysterectomy for cervical cancer, for a number of surgeons the question remains whether a minimally invasive approach could potentially be considered for patients with small tumors $(<2 \mathrm{~cm}){ }^{8}{ }^{8}$ Recently, a series of retrospective studies have attempted to answer this question. However, 


\section{Original research}

given the low incidence of cervical cancer, the majority of studies do not have sufficient statistical power to detect a difference in progression-free survival among patients with small tumors, and in many studies follow-up of the minimally invasive group was short. ${ }^{9-24}$ The aim of the present systematic review and metaanalysis was to compare the reported oncologic outcomes between open and minimally invasive radical hysterectomy for patients with apparent early-stage cervical carcinoma and tumor size $\leq 2 \mathrm{~cm}$.

\section{METHODS}

This systematic review was conducted in accordance with the Preferred Reporting Items for Systematic Review and MetaAnalyses guidelines ${ }^{25}$; a protocol was determined beforehand by all participating authors and submitted for registration to the PROSPERO Registry (CRD42020207971). A comprehensive electronic search (from conception to end-of-search date January 30, 2021) of the Pubmed/Medline, Embase, and Web of Science databases was performed using the following keywords: (cervical cancer OR cervix) AND (hysterectomy or radical) AND (laparoscopy OR robotic OR minimally invasive $\mathrm{OR}$ laparoscopes $\mathrm{OR}$ laparotomy $\mathrm{OR}$ open); in addition, MESH terms were used. Reference lists of the included studies as well as prior systematic reviews were systematically hand searched. Observational studies published as full papers in English were eligible for inclusion. Inclusion criteria were: patients with invasive squamous, adenosquamous, or adenocarcinoma of the cervix; patients undergoing primary radical hysterectomy with lymphadenectomy for apparent early stage (FIGO 2009 stage IAIIA) disease; comparison of open and minimally invasive surgical techniques; studies with at least $(n=50)$ patients in each study group; follow-up of at least 24 months; studies reporting the number of patients with tumors $<=2 \mathrm{~cm}$ in each surgical modality group; studies reporting data on progression-free survival; studies that attempted to control survival for confounders known to be associated with survival, such as lymphovascular invasion, depth of invasion, age, presence of co-morbidities, and histology, with statistical methods such as Cox regression, propensity score matching, or inverse probability weighting. Exclusion criteria were: studies not reporting the number of patients with tumor size $\leq 2 \mathrm{~cm}$; performance of laparoscopic-assisted vaginal hysterectomy; significant overlapping population; no data on progression-free survival; administration of neo-adjuvant chemotherapy or radiation therapy; follow-up <24 months; not controlling for important confounders; no data on tumor size.

In this study we opted to select as primary outcome progressionfree survival while secondary outcomes were overall survival and location of relapse. The methodological quality of observational studies was assessed using the Newcastle-Ottawa Quality Assessment Scale for non-randomized studies. ${ }^{26}$ The scale was developed to assess the quality of non-randomized studies directed to the task of incorporating the quality assessments in the interpretation of meta-analytic results. Each study is evaluated on three broad perspectives: the selection of the study groups; the comparability of the groups; and the ascertainment of outcome of interest. Data extraction from all eligible papers was performed by two authors (DN, BBA) working independently. Any discrepancies were resolved by discussion. From each eligible study the following parameters were extracted when applicable: general study characteristics (author, country of origin, date of publication, years of recruitment), patient demographics, type of minimally invasive surgery (laparoscopic or robotic-assisted), number of relapses and deaths, progression-free (defined as time between diagnosis or surgery until first relapse), and overall survival (time from diagnosis or surgery to death from any cause). If the required data for the primary outcome were not readily available in the published articles or could not be extracted from published tables, online supplemental material or KaplanMeier curves, the corresponding authors were contacted. In studies with overlapping populations, the study with the largest number of patients was included.

The level of statistical heterogeneity was evaluated with the $\mathrm{X}^{2}$ and $\mathrm{I}^{2}$ statistic; values of $25 \%, 50 \%$, and $75 \%$ were considered low, moderate, and high heterogeneity, respectively A random-effects model was used to compare relapse and death rate between the two groups and $\mathrm{OR}$ and $95 \%$ confidence intervals were calculated with the Der Simonian and Laird approach. HR and 95\% confidence intervals were pooled using the generic inverse variance method as provided by the RevMan software. Forest plots were created for each comparison, while graphical funnel plots were generated to determine the presence of publication bias by visual inspection. $\mathrm{P}$ values $<0.05$ were considered statistically significant. If significant heterogeneity was noted, a sensitivity analysis was performed by a sequential omission algorithm. ${ }^{27}$ If $\mathrm{HR}$ and $95 \%$ confidence intervals were available, validated statistical methodology was used to generate them. ${ }^{28}$ Statistical analysis was performed with the Cochrane Review software (Review Manager version 5.2). ${ }^{29}$ This study did not require evaluation by an institutional review board.

\section{RESULTS}

A total of 10 studies that met the inclusion criteria were identified. ${ }^{10-13151620-22 ~ 24}$ The study selection flowchart is depicted in online supplemental Figure 1 while graphical funnel plot is depicted in online supplemental Figure 2. The majority of studies $(n=6)$ originated from Asia ${ }^{121315162122}$ followed by North America $(n=2),{ }^{1011}$ Europe $(n=1),{ }^{20}$ and one international study ${ }^{24}$ was identified. A total of 4935 patients were included, 2394 (48.5\%) patients had minimally invasive and 2541 (51.5\%) patients had open radical hysterectomy, respectively, for tumors with size $\leq 2 \mathrm{~cm}$. Table 1 summarizes the basic characteristics, median follow-up, and method of tumor size assessment in each of the included studies; online supplemental Table 1 summarizes clinicopathological characteristics from studies with available data. ${ }^{12} 15162122$ In nine studies, minimally invasive radical hysterectomy was performed with traditional laparoscopy while in two studies, originating from the United States, the vast majority of patients underwent robotic-assisted radical hysterectomy. The majority of studies $(n=8)$ were deemed of good quality with a total Newcastle-0ttowa score $\geq 7$ (Table 1).

Patients who underwent minimally invasive hysterectomy had worse progression-free survival than those who had open surgery (HR 1.68, 95\% Cl 1.20 to 2.36, I 26\%) (Figure 1). Pooled HR following serial exclusion of each study is presented in (online supplemental Table 2). When including studies $(n=7)^{13151620-22 ~} 24$ that reported at least 100 patients in each arm, the minimally invasive $(n=2100)$ group had worse progression-free survival than the open $(n=2216)$ 


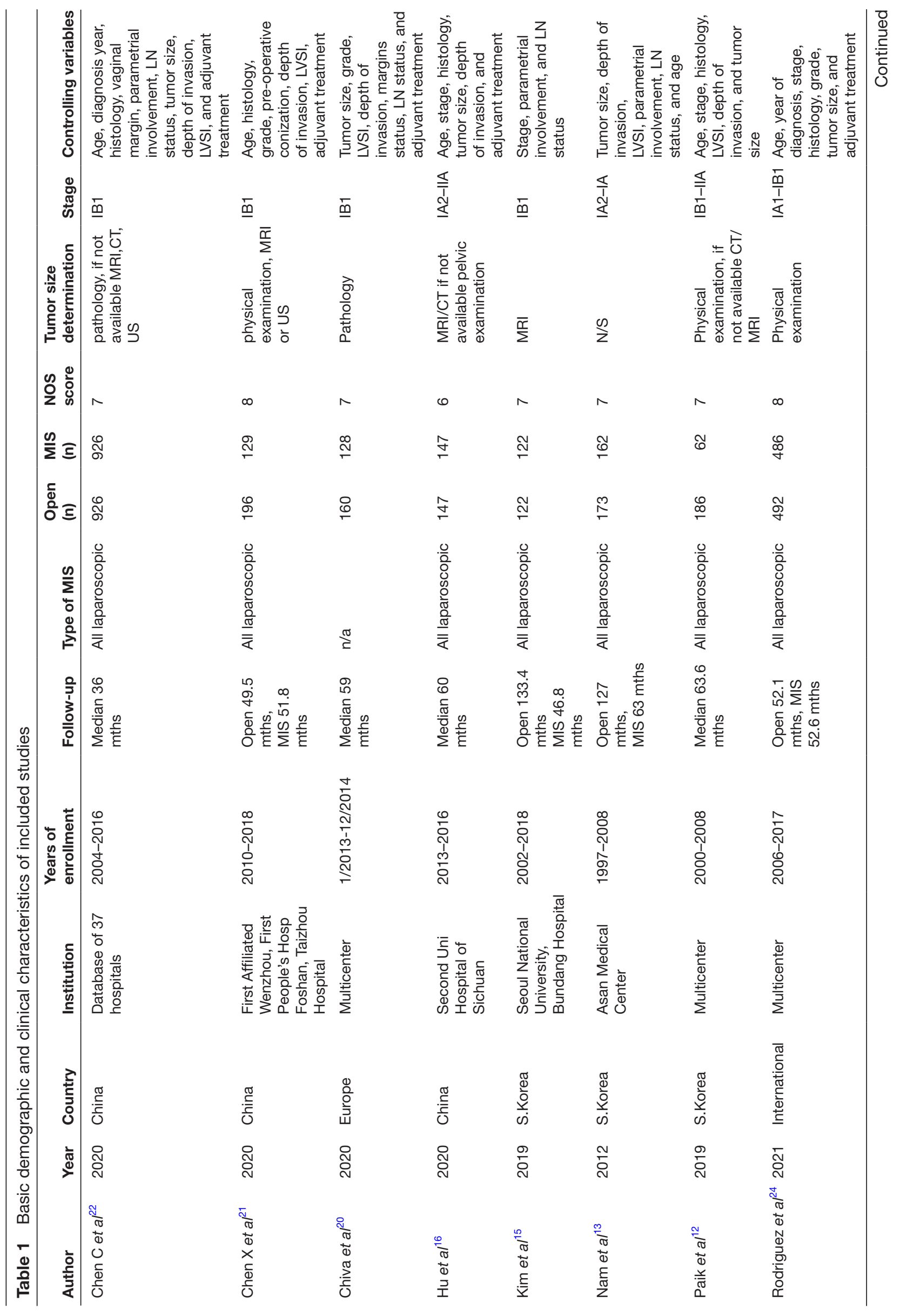


hysterectomy group (HR $1.50,95 \% \mathrm{Cl} 1.15$ to $1.96, \mathrm{I}^{2} 0 \%$ ). When including studies that determined tumor size based exclusively on pathology $(n=4)$ the minimally invasive $(n=1286)$ group, was associated with worse progression-free survival than the open $(\mathrm{n}=1225)$ hysterectomy group (HR $1.66,95 \% \mathrm{Cl} 1.16$ to $2.36, \mathrm{I}^{2}$ $0 \%$ ). When including studies that determined tumor size based on imaging and/or physical examination (clinical stage) exclusively $(n=5)$, there was no difference in progression-free survival between the minimally invasive $(n=946)$ and open $(n=1143)$ hysterectomy groups (HR $1.70,95 \% \mathrm{Cl} 0.84$ to $3.44, I^{2} 55 \%$ ). Based on data from studies originating from Asia $(n=6),{ }^{12} 1315162122$ performance of minimally invasive hysterectomy was not associated with worse progression-free survival (HR 1.73, 95\% $\mathrm{Cl} 0.99$ to $3.03, \mathrm{I}^{2} 46 \%$ ). Similarly, based on data from studies originating from Europe and the Americas $(n=4),{ }^{10} 112024$ performance of minimally invasive hysterectomy was associated with worse progression-free survival (HR $1.65,95 \% \mathrm{Cl} 1.11$ to $2.47, \mathrm{I}^{2} 0 \%$ ).

Based on data from five studies, ${ }^{11} 16202224$ patients who had minimally invasive $(n=1808)$ hysterectomy had a trend towards worse overall survival compared with those who had open surgery $(\mathrm{n}=1853)$ (HR 1.64, 95\% Cl 1.00 to 2.68) (Figure 2). Table 2 summarizes the outcomes reported in each study. Data on the location of relapse were available from four studies, ${ }^{12} 152122$ and included 35 and 59 cases in the open and minimally invasive surgery groups (online supplemental Table 3). Among patients who had open surgery, $51.4 \%(n=18)$ experienced an abdominal/distant relapse, $8.6 \%(n=3)$ lymph node relapse, and $40 \%(n=14)$ a pelvic/vaginal relapse. For patients who had minimally invasive surgery, $64.4 \%$ $(n=38)$ had an abdominal/distant relapse, $3.4 \%(n=2)$ a lymph node relapse, and $30.5 \%(n=18)$ a pelvic/vaginal relapse.

\section{DISCUSSION}

\section{Summary of Main Results}

Based on a large number of patients, minimally invasive radical hysterectomy was associated with a worse progression-free survival than open surgery. Low heterogeneity was found but a series of sensitivity analyses (mode of tumor size assessment, country of origin, and number of participants) were performed to aid in the interpretation of our results. Patients who underwent minimally invasive radical hysterectomy also had worse overall survival and the difference approached statistical significance. Based on data from four studies, location of tumor relapse did not differ between the minimally invasive and open groups.

\section{Results in the Context of Published Literature}

Data on the oncologic safety of minimally invasive surgery for patients with cervical cancer and small tumors are heterogeneous and conflicting. A recent randomized trial did not find a higher relapse rate in the subgroup of patients with tumors, $<2 \mathrm{~cm}$, but it was not designed or powered to identify such a difference. ${ }^{4}$ In a retrospective study focusing exclusively on patients with tumors $<2 \mathrm{~cm}$, Chen et al reported better 5-year progression-free survival for patients who had open surgery $(n=196,97.7 \%)$ than for those who had minimally invasive radical hysterectomy $(n=129,90.4 \%)$, $\mathrm{p}=0.016 .{ }^{21}$ After controlling for confounders, laparoscopic approach was associated with worse disease-free survival $(\mathrm{HR} 4.64,95 \% \mathrm{Cl}$ 1.26 to 17.06$).{ }^{21}$ Similarly, Uppal et al performed a multicenter 


\begin{tabular}{|c|c|c|c|c|c|c|c|c|}
\hline Study or Subgroup & log[Hazard Ratio] & SE & $\begin{array}{l}\text { MIS } \\
\text { Total }\end{array}$ & $\begin{array}{l}\text { Open } \\
\text { Total }\end{array}$ & Weight & $\begin{array}{c}\text { Hazard Ratio } \\
\text { IV, Random, } 95 \% \mathrm{CI}\end{array}$ & $\begin{array}{r}\text { Hazard } \\
\text { IV, Randor }\end{array}$ & $\begin{array}{l}\text { Ratio } \\
\mathrm{m}, 95 \% \mathrm{Cl}\end{array}$ \\
\hline Chen C et al, 2020 & 0.4121 & 0.2206 & 926 & 926 & $25.1 \%$ & $1.51[0.98,2.33]$ & & $-\infty$ \\
\hline Chen $X$ et al, 2020 & 1.5347 & 0.6643 & 129 & 196 & $5.8 \%$ & $4.64[1.26,17.06]$ & & \\
\hline Chiva et al, 2020 & 0.4886 & 0.3695 & 128 & 160 & $14.3 \%$ & $1.63[0.79,3.36]$ & & 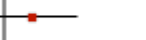 \\
\hline Hu et al, 2020 & -0.3425 & 0.5677 & 147 & 147 & $7.5 \%$ & $0.71[0.23,2.16]$ & & \\
\hline Kim et al, 2019 & 0.0526 & 0.4893 & 122 & 122 & $9.5 \%$ & $1.05[0.40,2.75]$ & & \\
\hline Nam et al, 2012 & 0.7747 & 0.5629 & 162 & 173 & $7.6 \%$ & $2.17[0.72,6.54]$ & & \\
\hline Paik et al, 2019 & 2.5639 & 1.1182 & 62 & 186 & $2.2 \%$ & $12.99[1.45,116.23]$ & & \\
\hline Rodriguez et al 2021 & 0.3646 & 0.2712 & 486 & 492 & $20.7 \%$ & $1.44[0.85,2.45]$ & & - \\
\hline Uppal et al, 2020 & 1.8421 & 0.8301 & 182 & 82 & $3.9 \%$ & $6.31[1.24,32.11]$ & & \\
\hline Yang et al 2020 & 0.5277 & 0.8938 & 50 & 57 & $3.4 \%$ & $1.70[0.29,9.77]$ & & \\
\hline Total $(95 \% \mathrm{Cl})$ & & & 2394 & 2541 & $100.0 \%$ & $1.68[1.20,2.36]$ & & \\
\hline \multicolumn{7}{|c|}{$\begin{array}{l}\text { Heterogeneity: } \operatorname{Tau}^{2}=0.07 ; \mathrm{Chi}^{2}=12.10, \mathrm{df}=9(\mathrm{P}=0.21) ; \mathrm{I}^{2}=26 \% \\
\text { Test for overall effect: } \mathrm{Z}=3.05(\mathrm{P}=0.002)\end{array}$} & $0.1{ }^{1}$ & Favours op \\
\hline
\end{tabular}

Figure 1 Pooled progression-free survival between minimally invasive and open radical hysterectomy groups. MIS, minimally invasive surgery.

retrospective study among institutions in the United States and Canada and reported inferior progression-free survival among 264 patients with tumors $<2 \mathrm{~cm}$ who had minimally invasive surgery. ${ }^{10}$

On the other hand, two recent analyses of large European nationwide databases did not find an increased risk of relapse for patients undergoing a minimally invasive approach. Alfonso et al examined the Swedish National Cancer Registry for patients with cervical carcinoma undergoing primary radical hysterectomy at high-volume centers and reported a 5-year progression-free survival rate of $92 \%$ in the open $(n=150)$ and $91 \%$ in the robotic-assisted $(n=460)$ group. ${ }^{30}$ Similarly, in an analysis of the Netherlands Cancer Registry that included 434 patients with tumors of $<2 \mathrm{~cm}$ who underwent radical hysterectomy between 2010 and 2017, there was no difference in progression-free survival between the open and minimally invasive groups; with 5-year progression-free survival rates of $91.4 \%$ and $96.4 \%$, respectively. ${ }^{9}$ Nevertheless, in both studies, survival was not controlled for important confounders, exclusively among patients with tumor size $<2 \mathrm{~cm}$. Another multicenter European retrospective study (SUCCOR) that recruited patients from 29 countries and 126 institutions, and controlled for important confounders did not find a significantly worse progression-free survival between the minimally invasive $(n=126)$ and open $(n=241)$ groups (HR $1.63,95 \% \mathrm{Cl} 0.79$ to 3.40$){ }^{20}$

A critical point should be underlined; in several studies reported in the literature, patients who underwent open hysterectomy had significantly longer follow-up, while many studies do not control for confounders such as histology, presence of lymphovascular invasion, and post-operative treatment. A shorter follow-up can confound results since late relapses are not captured, while differences in overall survival are not evident. Similar to our results, in a recent analysis of the National Cancer Database, that included 2046 patients with cervical carcinoma and tumor size $\leq 2 \mathrm{~cm}$ who underwent minimally invasive $(n=1195)$ or open $(n=851)$ radical hysterectomy, those who had a minimally invasive approach had a worse overall survival ( $\mathrm{HR} 1.72,95 \% \mathrm{Cl} 1.05$ to 2.82) after controlling for confounders. ${ }^{31}$ However, that study was limited by the lack of central pathology review, data on surgeon's experience, and cause of death.

The etiology of increased relapse among those receiving minimally invasive surgery is a matter of debate. One theory is that use of an uterine manipulator may be associated with intraperitoneal spread of cancer cells. In a recent multi-institutional study, patients who underwent minimally invasive surgery without the use of an uterine manipulator had similar rate of relapse to those who had an open surgery (HR 1.58; $95 \% \mathrm{Cl} 0.79$ to $3.15 ; p=0.20$ ), while within the minimally invasive surgery group use of a uterine manipulator was associated with higher relapse rate. ${ }^{20}$ However, in an analysis of 224 patients who underwent minimally invasive radical hysterectomy at two large Canadian centers, after controlling for confounders, use of a uterine manipulator was not associated with recurrence risk. ${ }^{32}$ Lack of appropriate surgical expertise may also contribute to worse oncologic outcomes. Chong et al reported the surgical and oncologic outcomes of 100 consecutive patients. With improving surgical experience, operating time and the perioperative complication rate decreased, but overall and progressionfree survival remained the same. ${ }^{33} \mathrm{Kim}$ et al analyzed the learning curve of a single surgeon and reported poorer progression-free survival during the early phase of minimally invasive surgery. ${ }^{34}$

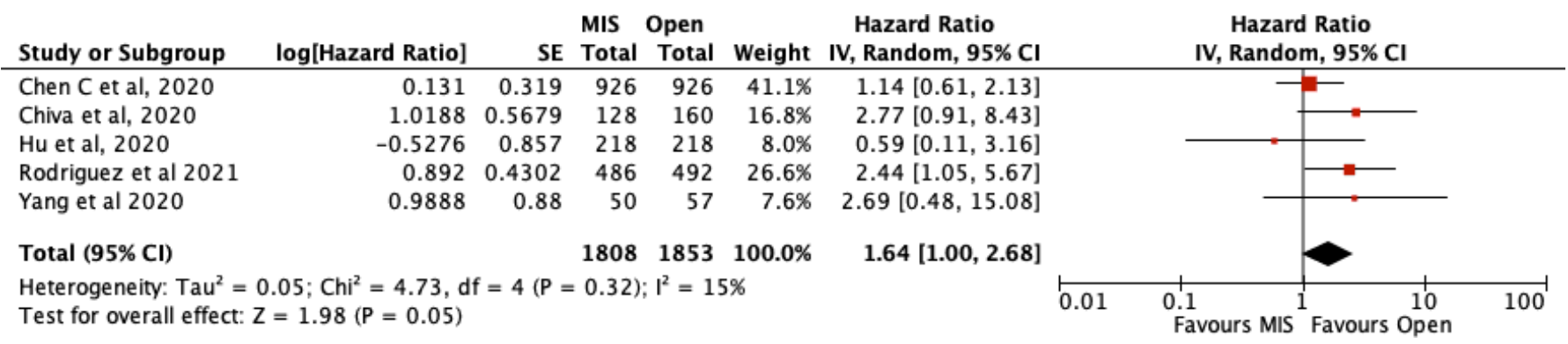

Figure 2 Pooled overall survival between minimally invasive and open radical hysterectomy groups. MIS, minimally invasive surgery. 


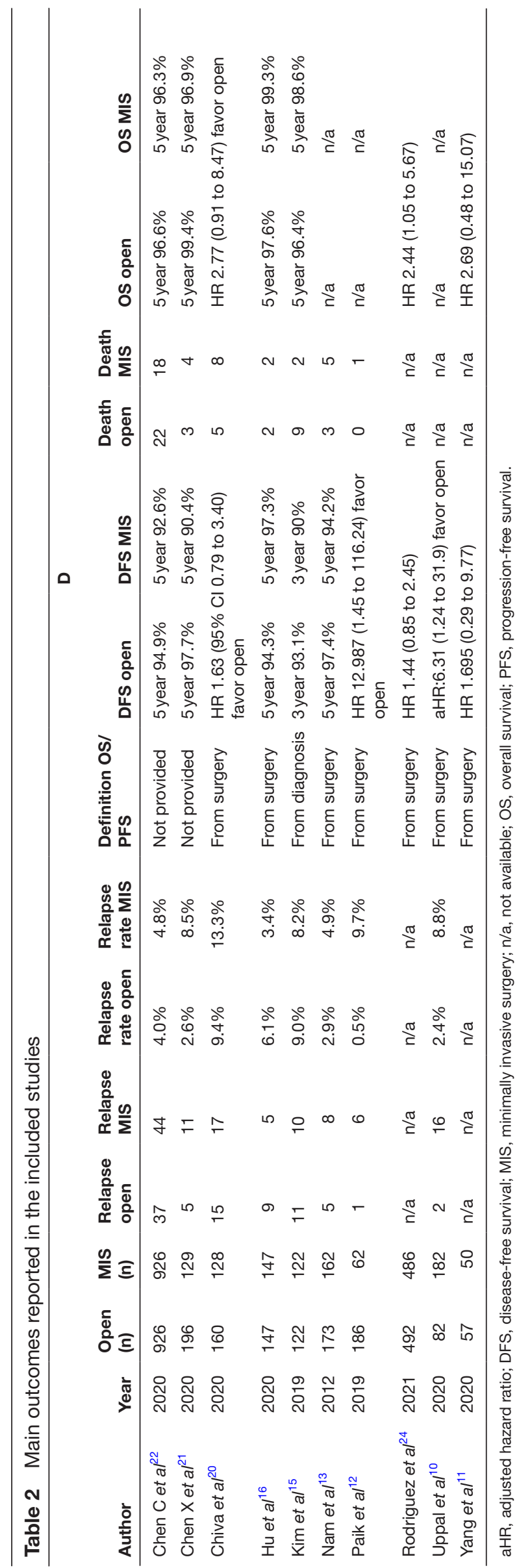

We should underline that pre-operative tumor size assessment by physical examination and imaging is challenging. ${ }^{35}$ The majority of studies included in the present review, determined tumor size based on pathology report and, if not available, imaging or physical examination. Uppal et al reported significant discrepancies between pre-operative and pathologic tumor size; from 291 patients deemed as having no visible disease, $19.9 \%$ actually had tumors $>2 \mathrm{~cm}$ on final pathology while $34.6 \%$ of 257 patients with tumors $<2 \mathrm{~cm}$ based on pre-operative assessment had tumors $>2 \mathrm{~cm}$ on final pathology..$^{10}$ Thus selecting a specific tumor size cut-off point to establish the safety of a minimally invasive approach could be problematic. Nevertheless, a question that merits further investigation is whether a minimally invasive approach is safe for patients with documented microscopic tumors (stage IA2 disease) on physical examination and imaging, or no visible disease following cold knife cone. The number of patients with IA2 disease reported by retrospective studies is very small, while they have a baseline low relapse rate that would require a collaborative effort to obtain a considerable number of patients for a meaningful analysis. For patients who underwent conization and had no residual disease on pre-operative assessment, Uppal et al reported a relapse rate of $1.4 \%$ and $2.9 \%$ in the open $(n=72)$ and minimally invasive $(n=171)$ groups, $p=0.48 .{ }^{10}$ Casarin et al also identified 186 stage IA1-IB1 patients who underwent minimally invasive radical hysterectomy and reported that pre-operative conization was associated with a lower risk of relapse $(1.1 \%$ vs $16.1 \%, p<0.001)$ even for patients with stage IB1 disease $(1.8 \%$ vs $17.2 \%, p=0.004){ }^{36}$ Further research, is warranted to investigate whether minimally invasive surgery could be considered for patients with microscopic tumors or no visible disease following cold knife cone.

\section{Strengths and Weaknesses}

A major strength of the present study is the inclusion of a large number of patients with tumor size $\leq 2 \mathrm{~cm}$ who underwent minimally invasive or open radical hysterectomy. We performed a thorough review of the literature and a series of sensitivity analyses that can aid readers in the interpretation of our results, while the Newcastle-Ottawa scale was used to evaluate the quality of the included studies. However, certain limitations should be mentioned. First, although all studies controlled for important confounders, post-operative treatment and surveillance might have varied. Only one study reported the performance of protective maneuvers to prevent tumor contamination, and thus its impact on relapse rate could not be assessed in a dedicated sub-analysis. ${ }^{20}$ Similarly, evaluation of relapse rate among patients with no residual disease following cone biopsy could not be performed since only two studies reported such an outcome. ${ }^{10}$ Moreover, not all studies provided the exact method of tumor size determination while some used a combination of imaging, physical examination, and pathology report. When imaging was used, authors did not clarify how many patients underwent an MRI versus other imaging modalities, such as CT or ultrasound, which may be less sensitive in tumor measurement. In addition, relapse rate in each study varied, probably secondary to patient risk factors, variation in surgical expertise, and post-operative adjuvant treatment. We opted to include studies that had a minimum of 24 months of follow-up in each arm, similar to a recent meta-analysis, ${ }^{6}$ though as previously discussed, follow-up might not have captured all relapses, especially in the minimally 
invasive hysterectomy groups. Lastly, we excluded studies that did not report any data on tumor size, since we could not request authors to individually collect additional data and re-analyze them. Thus some large retrospective studies did not meet our inclusion criteria. ${ }^{37}{ }^{38}$ Also, only one of the included studies provided data on blood loss, intra-operative and post-operative complications and so we could not compare differences in peri-operative outcomes between the two groups. Since there was a possibility of some minor overlap between two studies, ${ }^{20}{ }^{24}$ we performed a sensitivity analysis excluding serially each study, and our results did not change.

\section{Implications for Practice and Future Research}

Given the accumulating evidence demonstrating worse oncologic outcomes for patients undergoing minimally invasive radical hysterectomy, and difficulty in accurately assessing tumor size preoperatively, an open approach should be selected. An international effort to perform individual patient data meta-analysis with information on important confounders and post-operative treatment could aid in further elucidating outcomes of minimally invasive surgery for patients with microscopic or small tumors or no residual disease.

\section{CONCLUSIONS}

In this systematic review of literature and meta-analysis that compiled data from a large number of patients who underwent primary radical hysterectomy with tumor size $\leq 2 \mathrm{~cm}$, minimally invasive radical hysterectomy was associated with worse progression-free survival.

\section{Twitter Benjamin B Albright @BenAlbrightMD}

Acknowledgements We would like to thank Dr Brown, Dr Chiva, Dr Gil-Moreno, and Dr Wenzel for providing additional information from their published papers.

Contributors DN: Conceptualization; data curation; statistical analysis; formal analysis; investigation; methodology; project administration; resources; software; visualization; writing - original draft; writing - review and editing. BBA: Data curation; formal analysis; investigation; methodology; project administration; resources; software; visualization; writing - original draft; writing - review and editing. SK, MM, RLG II, AFH, EMK: Investigation; methodology; writing - original draft; writing - review and editing. NL: Supervision; formal analysis; investigation; methodology; writing - original draft; writing - review and editing.

Funding The authors have not declared a specific grant for this research from any funding agency in the public, commercial or not-for-profit sectors.

Competing interests None declared.

Patient consent for publication Not required.

Provenance and peer review Not commissioned; externally peer reviewed.

Data availability statement Data are available upon reasonable request.

Supplemental material This content has been supplied by the author(s). It has not been vetted by BMJ Publishing Group Limited (BMJ) and may not have been peer-reviewed. Any opinions or recommendations discussed are solely those of the author(s) and are not endorsed by BMJ. BMJ disclaims all liability and responsibility arising from any reliance placed on the content. Where the content includes any translated material, BMJ does not warrant the accuracy and reliability of the translations (including but not limited to local regulations, clinical guidelines, terminology, drug names and drug dosages), and is not responsible for any error and/or omissions arising from translation and adaptation or otherwise.

\section{ORCID iDs}

Dimitrios Nasioudis http://orcid.org/0000-0001-6260-5353

Benjamin B Albright http://orcid.org/0000-0001-5296-9575

\section{REFERENCES}

1 Sung H, Ferlay J, Siegel RL, et al. Global cancer statistics 2020: GLOBOCAN estimates of incidence and mortality worldwide for 36 cancers in 185 countries. CA Cancer J Clin 2021. doi:10.3322/ caac.21660. [Epub ahead of print: 04 Feb 2021].

2 Abu-Rustum NR, Yashar CM, Bean S, et al. NCCN guidelines insights: cervical cancer, version 1.2020. J Natl Compr Canc Netw 2020;18:660-6.

3 Melamed A, Rauh-Hain JA, Ramirez PT. Minimally invasive radical hysterectomy for cervical cancer: when adoption of a novel treatment precedes prospective, randomized evidence. J Clin Oncol 2019;37:3069-74.

4 Ramirez PT, Frumovitz M, Pareja R, et al. Minimally invasive versus abdominal radical hysterectomy for cervical cancer. $N$ Engl J Med 2018;379:1895-904.

5 Melamed A, Margul DJ, Chen L, et al. Survival after minimally invasive radical hysterectomy for early-stage cervical cancer. $N$ Engl J Med 2018;379:1905-14.

6 Nitecki R, Ramirez PT, Frumovitz M, et al. Survival after minimally invasive vs open radical hysterectomy for early-stage cervical cancer: a systematic review and meta-analysis. JAMA Oncol 2020;6:1019-27.

7 Uppal S, Spencer R. Modify or abandon: minimally invasive radical hysterectomy for early-stage cervical cancer. Int J Gynecol Cancer 2019;29:843-4

8 Greggi S, Casella G, Scala F, et al. Surgical management of early cervical cancer: when is laparoscopic appropriate? Curr Oncol Rep 2020;22:7.

9 Wenzel HHB, Smolders RGV, Beltman JJ, et al. Survival of patients with early-stage cervical cancer after abdominal or laparoscopic radical hysterectomy: a nationwide cohort study and literature review. Eur J Cancer 2020;133:14-21.

10 Uppal S, Gehrig PA, Peng K, et al. Recurrence rates in patients with cervical cancer treated with abdominal versus minimally invasive radical hysterectomy: a multi-institutional retrospective review study. $J$ Clin Oncol 2020;38:1030-40.

11 Yang J, Mead-Harvey C, Polen-De C, et al. Survival outcomes in patients with cervical cancer treated with open versus robotic radical hysterectomy: our surgical pathology interrogation. Gynecol Oncol 2020;159:373-80.

12 Paik ES, Lim MC, Kim M-H, et al. Comparison of laparoscopic and abdominal radical hysterectomy in early stage cervical cancer patients without adjuvant treatment: ancillary analysis of a Korean Gynecologic Oncology Group study (KGOG 1028). Gynecol Oncol 2019;154:547-53.

13 Nam J-H, Park J-Y, Kim D-Y, et al. Laparoscopic versus open radical hysterectomy in early-stage cervical cancer: long-term survival outcomes in a matched cohort study. Ann Oncol 2012;23:903-11.

14 Levine MD, Brown J, Crane EK, et al. Outcomes of minimally invasive versus open radical hysterectomy for early stage cervical cancer incorporating 2018 FIGO staging. J Minim Invasive Gynecol 2021;28:S1553-4650(20)30352-6

$15 \mathrm{Kim}$ SI, Lee M, Lee S, et al. Impact of laparoscopic radical hysterectomy on survival outcome in patients with FIGO stage lb cervical cancer: a matching study of two institutional hospitals in Korea. Gynecol Oncol 2019;155:75-82.

16 Hu TWY, Huang Y, Li N, et al. Comparison of laparoscopic versus open radical hysterectomy in patients with early-stage cervical cancer: a multicenter study in China. Int J Gynecol Cancer 2020;30:1143-50.

17 Gil-Moreno A, Carbonell-Socias M, Salicrú S, et al. Radical hysterectomy: efficacy and safety in the dawn of minimally invasive techniques. J Minim Invasive Gynecol 2019;26:492-500.

18 Doo DW, Kirkland CT, Griswold LH, et al. Comparative outcomes between robotic and abdominal radical hysterectomy for IB1 cervical cancer: results from a single high volume institution. Gynecol Oncol 2019;153:242-7.

19 Li P, Chen L, Ni Y, et al. Comparison between laparoscopic and abdominal radical hysterectomy for stage IB1 and tumor size $<2$ $\mathrm{cm}$ cervical cancer with visible or invisible tumors: a multicentre retrospective study. J Gynecol Oncol 2021;32:e17.

20 Chiva L, Zanagnolo V, Querleu D, et al. SUCCOR study: an international European cohort observational study comparing minimally invasive surgery versus open abdominal radical hysterectomy in patients with stage IB1 cervical cancer. Int $J$ Gynecol Cancer 2020;30:1269-77.

21 Chen X, Zhao N, Ye P, et al. Comparison of laparoscopic and open radical hysterectomy in cervical cancer patients with tumor size $\leq 2$ cm. Int J Gynecol Cancer 2020;30:564-71.

22 Chen C, Liu P, Ni Y, et al. Laparoscopic versus abdominal radical hysterectomy for stage IB1 cervical cancer patients with tumor 


\section{Original research}

size $\leq 2 \mathrm{~cm}$ : a case-matched control study. Int $\mathrm{J}$ Clin Oncol 2020;25:937-47.

23 Brandt B, Sioulas V, Basaran D, et al. Minimally invasive surgery versus laparotomy for radical hysterectomy in the management of early-stage cervical cancer: survival outcomes. Gynecol Oncol 2020;156:591-7.

24 Rodriguez J, Rauh-Hain JA, Saenz J, et al. Oncological outcomes of laparoscopic radical hysterectomy versus radical abdominal hysterectomy in patients with early-stage cervical cancer: a multicenter analysis. Int J Gynecol Cancer 2021;31:-504-11.

25 Liberati A, Altman DG, Tetzlaff J, et al. The PRISMA statement for reporting systematic reviews and meta-analyses of studies that evaluate healthcare interventions: explanation and elaboration. BMJ 2009;339:b2700.

26 Wells GA SB, O'Connell D, Peterson J. The Newcastle-Ottawa scale (NOS) for assessing the quality of non randomized studies in metaanalyses. Ottawa, Canada: Dept of Epidemiologyand Community Medicine, University of Ottawa, 2014.

27 Patsopoulos NA, Evangelou E, loannidis JPA. Sensitivity of betweenstudy heterogeneity in meta-analysis: proposed metrics and empirical evaluation. Int J Epidemiol 2008;37:1148-57.

28 Tierney JF, Stewart LA, Ghersi D, et al. Practical methods for incorporating summary time-to-event data into meta-analysis. Trials 2007;8:16.

29 The Nordic Cochrane Centre, The Cochrane Collaboration. Review Manager (RevMan) [Computer program. Version 5.3. Copenhagen, 2014.

30 Alfonzo E, Wallin E, Ekdahl L, et al. No survival difference between robotic and open radical hysterectomy for women with early-stage cervical cancer: results from a nationwide population-based cohort study. Eur J Cancer 2019;116:169-77.
31 Nasioudis D, Albright BB, Haggerty AF, et al. Survival following minimally invasive radical hysterectomy for patients with cervical carcinoma and tumor size $\leq 2 \mathrm{~cm}$. Am J Obstet Gynecol 2021;224:317-8.

32 Nica A, Kim SR, Gien LT, et al. Survival after minimally invasive surgery in early cervical cancer: is the intra-uterine manipulator to blame? Int J Gynecol Cancer 2020;30:1864-70.

33 Chong GO, Lee YH, Lee HJ, et al. Comparison of the longterm oncological outcomes between the initial learning period of robotic and the experienced period of laparoscopic radical hysterectomy for early-stage cervical cancer. Int J Gynecol Cancer 2018;28:226-32.

$34 \mathrm{Kim} \mathrm{S}$, Min KJ, Lee S, et al. Learning curve could affect oncologic outcome of minimally invasive radical hysterectomy for cervical cancer. Asian J Surg 2021;44:174-80.

35 Salvo G, Odetto D, Saez Perrotta MC, et al. Measurement of tumor size in early cervical cancer: an ever-evolving paradigm. Int $J$ Gynecol Cancer 2020;30:1215-23.

36 Casarin J, Bogani G, Papadia A, et al. Preoperative conization and risk of recurrence in patients undergoing laparoscopic radical hysterectomy for early stage cervical cancer: a multicenter study. $J$ Minim Invasive Gynecol 2021;28:117-23.

37 Shah CA, Beck T, Liao JB, et al. Surgical and oncologic outcomes after robotic radical hysterectomy as compared to open radical hysterectomy in the treatment of early cervical cancer. J Gynecol Oncol 2017;28:e82.

38 Cusimano MC, Baxter NN, Gien LT, et al. Impact of surgical approach on oncologic outcomes in women undergoing radical hysterectomy for cervical cancer. Am J Obstet Gynecol 2019;221:619.e1-619.e24 